\title{
Usages Et Vulnerabilite De Pterocarpus Santalinoides L'her. Ex De (Papillionoidae), Une Plante Utilisee Dans Le Traitement Des Gastro-Enterites Dans Le Sud Du Benin
}

\author{
Ayena A C. \\ Agassounon Djikpo Tchibozo M.
}

Faculté des Sciences et Techniques, Université d'Abomey-Calavi, Bénin, Laboratoire des Normes et de Contrôle de qualités Microbiologique, Nutritionnelle et Pharmacologique/Laboratoire de Génétique et des Biotechnologies, RP Cotonou Assogbadjo A. E.

Laboratoire d'Ecologie Appliquée, Faculté des Sciences Agronomique, Université d'Abomey-Calavi, Bénin, Cotonou, Bénin

\section{Adoukonou-Sagbadja $\mathrm{H}$.}

Faculté des Sciences et Techniques, Université d’Abomey-Calavi, Bénin, Laboratoire des Normes et de Contrôle de qualités Microbiologique,

Nutritionnelle et Pharmacologique/Laboratoire de Génétique et des

Biotechnologies, RP Cotonou

\section{Mensah G. A}

Institut National des Recherches Agricoles du Bénin (INRAB), RP Cotonou

\section{Agbangla $C$. \\ Ahanhanzo C.}

Faculté des Sciences et Techniques, Université d’Abomey-Calavi, Bénin,

Laboratoire des Normes et de Contrôle de qualités Microbiologique,

Nutritionnelle et Pharmacologique/Laboratoire de Génétique et des Biotechnologies, RP Cotonou

doi: 10.19044/esj.2016.v12n6p218 URL:http://dx.doi.org/10.19044/esj.2016.v12n6p218

Abstract

In West Africa, rural populations depend heavily on woody plant resources to satisfy particular nutritional and therapeutic needs. This study was conducted in South of Benin to identify local knowledge about Pterocarpus santalinoides, and its vulnerability level. Investigations were made using an interview guide followed by observations. 180 professionals were interviewed. It appears from the study that the "African teak" is known 
as 10 local designations. The species is sought in many areas of use (African medicine, food medicine, carpentry, art, energy and well-being). On medicinal plan, leaves, bark of the trunk and roots are solicited alone or in association with others to treat especially the symptoms related to gastroenteric (diarrhoea, dysentery, vomiting and abdominal cramps). Decoct is the main galenic form adopted for the treatment of these conditions. Considering all the sectors, all its vegetative organs are used. Degree of uses of various organs of this plant is the main causes of its vulnerability. Vulnerability index (Iv) is equal to 2.4. The species is thus identified as vulnerable. It is urgent for its users to adopt a sustainable management approach, in order to preserve African teak.

Keywords: Pterocarpus santalinoides - African teak - Threat - Conservation

\section{Résumé}

En Afrique de l'Ouest, les populations rurales dépendent fortement des ressources végétales ligneuses pour satisfaire surtout leurs besoins alimentaires et thérapeutiques. Cette étude a été réalisée au Sud du Bénin dans le but de répertorier et de documenter les connaissances locales liées au « teck africain » (Pterocarpus santalinoides), et son niveau de vulnérabilité. Des enquêtes ont été faites à l'aide d'un guide d'entretien suivie des observations sur le terrain. 180 professionnels choisis de façon aléatoire ont été interviewés. Il ressort de l'étude, que le « teck africain » est connue sous 10 appellations locales. L’espèce est sollicitée dans sept secteurs d'usage (médecine traditionnelle, alimentaire, agroalimentaire, menuiserie, art, énergie domestique, bien-être). Sur le plan médicinal, les feuilles, les écorces du tronc et ses racines sont sollicités seuls ou en association avec d'autres pour traiter surtout les symptômes liés aux gastroentérites (diarrhées, dysenteries, vomissement et les crampes abdominales). Le décocté constitue la principale forme galénique adoptée pour le traitement de ces affections. En considérant l'ensemble des secteurs, tous ses organes végétatifs sont utilisés. Le degré de sollicitations des différents organes de cette plante se révèlent être les principales causes de sa vulnérabilité. L’indice de vulnérabilité (IV) est égal à 2,4. L’espèce est de ce fait identifiée comme vulnérable. Il est urgent pour ses utilisateurs, d'adopter dans une approche de gestion durable, des méthodes de conservation afin de préserver le teck africain.

Mots-clés : Pterocarpus santalinoides - Ethnobotanique - Menaces Conservation. 


\section{Introduction}

Les espèces forestières sont abondantes dans les écosystèmes naturelles ou formations reconstituées, qui à travers leurs Produits Forestiers Non Ligneux (PFNL), contribuent de façon significative à la santé humaine et à l'alimentation (Assogbadjo et al., 2005a, 2006). En dehors des plantes cultivées, plusieurs milliers de plantes sauvages peu connues revêtent une importance culturelle et un fort potentiel économique pour la médecine, l'alimentation, l'énergie, la construction et l'artisanat (Belem et Sanou,2009 ; Benkhnigue, 2011 ; Agassounon Djikpo Tchibozo et al., 2012 a,b ). Bien que les formations végétales et agro écosystèmes constituent une source et un réservoir potentiel de PFNL, peu de recherches ont été effectuées au Bénin sur ces produits qualifiés de «mineurs" (Codjia et al., 2003 ; Assogbadjo et al., 2005b ; Adoukonou-Sagbadja et al., 2006 ; Agassounon Djikpo Tchibozo et al., 2012b). Ces espèces à usage multiple sont souvent négligées par la nouvelle génération, entrainant de ce fait l'érosion du patrimoine végétale, la disparition des connaissances endogènes liées à leurs utilisations et leurs potentielles valorisantes. Des récentes études menées sur la connaissance et la perception des populations locales dans le Sahel ont permis non seulement de connaître de nombreuses utilisations des espèces ligneuses, mais aussi de comprendre l'ampleur des activités anthropiques sur la végétation (Hahn-Hadjali et Thiombiano, 2000; Kristensen et Balslev, 2003 ; Lykke et al., 2004 ; Wezel et Lykke, 2006 ; Adomou et al., 2011). Ces travaux ont révélé la rareté d'un certain nombre d'espèces Khaya senegalensis, Kigelia africana et Pterocarpus erinaceus. Si les facteurs anthropiques dégradant les ressources végétales ont été décrits largement dans la littérature, il existe très peu d'études sur la vulnérabilité des espèces (Betti, 2001 ; Traoré et al., 2011). La vulnérabilité correspond au degré d'exposition aux risques de réduction, de menace ou de disparition de certaines espèces végétales occasionnées par les modes de prélèvements inappropriés dans un environnement soumis à une pression anthropique croissante. Dès lors, l'évaluation de la vulnérabilité en prenant en compte les facteurs anthropiques constitue un impératif majeur de la réussite des actions de gestion durable des ressources végétales. Au Sud du Bénin, pour le traitement des gastroentérites, les populations font recours à plusieurs plantes médicinales dont Pterocarpus santalinoides L'Hér. ex De, de la famille des Papillionoidae, fait partie des plus citées et possède une excellente activité antibactérienne (Ayéna et al., 2014). La demande de plus en plus croissante des organes de cette ressource forestière pour traiter les gastroentérites, couplée à la taille de la population sans cesse grandissante au Sud du Bénin engendrent une augmentation de la pression anthropique exercée sur cette dernière. Cette étude a pour objectif principal de documenter les données endogènes sur tous les usages de $P$. santalinoides et d'évaluer son degré de 
vulnérabilité. Il s’est agi de : (i) répertorier les usages des organes de la plante ; (ii) les modes de récolte ; (iii) les facteurs de sa vulnérabilité.

\section{Zone d'étude}

L’étude a été conduite dans les Départements du Sud-Bénin auprès des professionnels de la médecine traditionnelle (tradithérapeutes, phytothérapeutes et herboristes) et autres personnes détenteurs de connaissances des Produits Forestiers Non Ligneux (PFNL). Le Sud-Bénin est caractérisé par un climat tropical humide ou un climat subéquatorial (béninien) à 4 saisons d’inégale durée, deux saisons de pluie alternant avec deux saisons sèches. La température moyenne annuelle varie de 25 à $29^{\circ} \mathrm{C}$.

\section{Détermination de la taille de l'échantillonnage}

Une pré-enquête exploratoire effectuée sur un échantillon de 80 personnes dans la région d'étude a montré que $50 \%$ des personnes interrogées connaissances et utilisent $P$. santalinoides dans divers applications. Cette donnée a été exploitée pour utiliser la formule de Dagnelie (1998) afin de déterminer la taille de l'échantillon total à enquêter. $\mathrm{N}=\frac{\mathrm{U}_{1-\alpha / 2}^{2} \mathrm{x}^{p(1-p)}}{\mathrm{d}^{2}}$; où $\mathrm{N}$ est la taille de l'échantillon considéré dans cette étude ; $U_{1-\alpha / 2}^{2}$ est la valeur de la variable aléatoire normale pour une valeur de la probabilité $\propto=0,05 ; U_{1-\alpha / 2}=1,96$; p est la proportion des personnes enquêtées lors de l'enquête exploratoire et qui sollicitent $P$. santalinoides pour leurs besoins $(p=0,5)$ et $d$ est la marge de l'erreur attendue. La valeur de "d" utilisée dans ce travail est arrondie à 0,08 . De ces hypothèses, la taille de l'échantillon égale à 176 personnes est arrondie à 180 pour les modèles pratiques de l'enquête. Les interviews ont été effectués dans les langues locales des enquêtés.

\section{Conduite des enquêtes de terrain}

Pour la réalisation des enquêtes proprement dite, la méthodologie d'enquête utilisée est celle rapportée par Mayling (1983), couplée à celle décrite par Bognon (1991). Il s'agit d'un face à face individuel au moyen d'un guide d'entretien suivie de la prise de notes ethnobotaniques. Vingtcinq personnes au moins des 2 sexes ont été contactées par Département et au total 180 professionnels choisis de façon aléatoire ont été interviewés. Puis des constats par observations directes sur le terrain ainsi que des recherches bibliographiques ont complété l'étude. Ce travail a duré 6 mois, avec un temps minimum d'une heure par rencontre. L'identification botanique des échantillons a été faite à l'Herbier National de l'Université d'Abomey-Calavi (Bénin). 


\section{Enquêtes socioculturelles et phytothérapie}

Des entretiens individuels et par groupe de 8 personnes au moins ont été faits. A chaque rencontre un échantillon de tige feuillée de $P$. santalinoides est présenté. Les informations collectées ont porté sur le profil socioculturel de l'enquêté (âge, niveau d'instruction et classe professionnelle), l’origine de la plante, les appellations (s) vernaculaire (s) ou locaux de $P$. santalinoides, les usages des différents organes de la plante (médicinales, nutritionnelles, construction, etc.). Pour limiter les erreurs dans le diagnostic des symptômes liés aux gastroentérites, le guide d'entretien a encore considéré des questions sur les symptômes déjà connus (Cuq, 2007). Par ailleurs, les questions ont concerné les maladies traitées et particulièrement les symptômes liés aux gastroentérites (diarrhée, dysenterie, vomissement, maux de ventre) et autres traitements dans lesquels les organes de la plante sont sollicités. La nature des aliments consommés avant les troubles est prise en compte. La fréquence et les heures d'apparition des symptômes après le repas. L'existence d'autres recettes associant cette plante pour traiter les gastroentérites et autres pathologies a été abordée ainsi que la source (achat, récolte) et/ou le mode de collecte des organes, le traitement fait avant l'usage des organes, les formes galéniques, la posologie, les techniques de conservation des phytomédicaments. Le stade de développement de l’organe utilisé ; la fréquence d'utilisation.

\section{Détermination des indices de vulnérabilités}

Les indices de vulnérabilité ont été calculés à partir des paramètres cités par Betti (2001) et par Traoré et al. (2011). Il s’agit des fréquences d'utilisation (U1) ; des types d'usage de l'espèce (U2). Les organes utilisés (U3) ; le mode (ramassage, cueillette et coupe) de prélèvement (U4) et le stade de développement de l’organe prélevé (U5) ont été considérés. Les stades de développement (jeune, adulte, vieux ou sénescent) retenus par Betti (2001), sont ceux utilisés dans le cadre de ce travail. Seule la partie qui a la plus grande valeur issue de l'échelle de vulnérabilité a été prise en compte pour le calcul des indices.

\section{Analyses des données}

La manière dont les données ethnobotaniques ont été traitées et analysées n’a pas été intégrée. Il en est de même pour la phytothérapie. Ceci est nécessaire pour la compréhension des résultats. La vulnérabilité de $P$. santalinoides a été déterminée à travers l'impact écologique de l'utilisation de ses organes. Les paramètres d'évaluation de vulnérabilité retenus sont ceux cités par Betti (2001), qu’est la fréquence de la popularité de l'espèce, des organes végétaux utilisés ; puis ceux évoqués par Traoré et al. (2011). La prise en compte de façon combinée des différents paramètres a permis de 
définir l’indice de vulnérabilité (Iv) pour l'espèce. Cet indice est obtenu en calculant la moyenne des différentes valeurs obtenues.. La fréquence d'utilisation (N1) d'une espèce i dans un usage j est calculée selon la formule suivante : $\mathrm{N} 1=$ npij/ntpe $\mathrm{x} 100$ avec npij= nombre de personnes ayant cité l'espèce $\mathrm{i}$ dans un usage $\mathrm{j}$; ntpe $=$ nombre total des informateurs. Les valeurs de N3, N4 et N5 varient de 1 à 3 suivant le type d'organe, le mode de prélèvement et le stade de développement de l’organe végétal. Ainsi, le calcul de l'indice de vulnérabilité de l'espèce (Iv ) suit la formule suivante : Iv $=\mathrm{N} / 5$ avec $\mathrm{N}=\mathrm{N} 1+\mathrm{N} 2+\mathrm{N} 3+\mathrm{N} 4+\mathrm{N} 5$. Si Iv $<2$, la plante est dite faiblement vulnérable; si $2 \leq \mathrm{Iv}<2,5$, la plante est dite moyennement vulnérable ; si Iv $\geq 2,5$, la plante est dite très vulnérable.

\section{Résultats}

\section{Caractéristiques sociodémographiques des professionnels}

Le profil et les caractéristiques socioculturelles sont présentés dans le tableau 1. Des enquêtes, il ressort que 100\% des personnes contactées ont une connaissance de l'espèce $P$. santalinoides (Figure 1).

Tableau 1 : Caractéristiques socioculturelles et profils des interviewés

\begin{tabular}{|c|c|c|c|c|c|c|c|c|c|c|}
\hline \multicolumn{11}{|c|}{ Caractéristiques socioculturelles } \\
\hline \multirow[t]{2}{*}{ Profils } & $\mathrm{Se}$ & & $\begin{array}{r}\text { Tran } \\
\text { d'â }\end{array}$ & & Tradithérapeutes & $\begin{array}{c}\text { Phyto- } \\
\text { thérapeutes }\end{array}$ & Herboristes & Autres & Instruits* & $\begin{array}{c}\text { Non } \\
\text { instruits }\end{array}$ \\
\hline & Hommes & Femmes & 25. 36 - $-x-3-$ & 66- & & 2 sex & xes confond & & & \\
\hline \multirow{2}{*}{ Effectifs } & 139 & 41 & $5 \quad 38$ & 137 & 79 & $\begin{array}{c}48 \\
\text { Ethnies }\end{array}$ & 36 & 17 & 40 & 140 \\
\hline & $\begin{array}{l}\mathrm{Fc} \\
6\end{array}$ & & $\begin{array}{c}\text { Adja } \\
46\end{array}$ & $\begin{array}{c}\text { Minc } \\
33\end{array}$ & $\begin{array}{c}\text { Gun } \\
27\end{array}$ & $\begin{array}{l}\text { Yorouba } \\
4\end{array}$ & $\begin{array}{c}\mathrm{Nag} \\
2\end{array}$ & & $\begin{array}{c}\text { Haoussa } \\
2\end{array}$ & $\begin{array}{l}\text { Igbo } \\
2\end{array}$ \\
\hline
\end{tabular}

Note : ${ }^{*}$ scolarisés niveau primaire (27); scolarisés niveau secondaire (13)

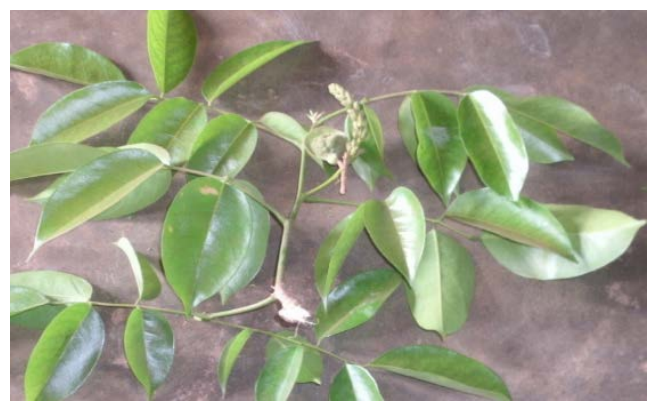

Figure 1 : Tige feuillée $P$. santalinoides avec les fleurs

\section{Appellations locales et différents usage des organes de $\boldsymbol{P}$. santalinoides}

L'espèce est désignée sous plusieurs noms vernaculaires selon les huit groupes socioculturels identifiés au Sud du Bénin (tableau 2). 
Tableau 2 : Désignations endogènes ou noms vernaculaires de $P$. santalinoides.

\begin{tabular}{cc}
\hline Groupes socioculturels & Noms vernaculaires de P. santalinoides \\
\hline Adja et Mina & Assouin, Baboutchi, Tonou-jiin, \\
Fon et Gun & Gbègbètin \\
Yorouba & Gbèngbèn \\
Nago & Ewèaègbè, Tigbi \\
Haoussa & Gunduru, Gyadarkurmi \\
Igbo & Uturukpa \\
\hline
\end{tabular}

\section{Divers usages de $P$. santalinoides}

Les résultats de l'enquête montrent que les populations à la base utilisent $P$. santalinoides dans sept secteurs à savoir la médecine africaine, l'alimentation humaine et animale, la menuiserie, l'agriculture, l'énergie, l'artisanat et le bien-être (Tableau 2). D'après les détenteurs des connaissances sur les PNLF l'espèce est exploitée dans les différents secteurs respectivement et proportionnellement à $52 \% ; 19 \% ; 13 \% ; 10 \%, 3 \%$ et $2 \% ; 1 \%$.

\section{Maladies traitées, organes utilisés et posologies}

L'enquête révèle que l'espèce est utilisée au Sud-Bénin dans le traitement de plusieurs affections. Il s'agit des : gastro-intestinales (dysenteries, diarrhées, vomissement, maux de ventre ou crampes abdominales); infections cutanées (variole et varicelle),respiratoires et sexuelles. 95\% des personnes contactées ont répondu spontanément que les feuilles ou les tiges feuillées de la plante traitent les gastroentérites (surtout les diarrhées et les dysenteries), les 5\% restant confirme l'utilisation des graines de petite taille (Ayéna et Agassounon Djikpo Tchibozo, 2015) pour cette affection. Parmi les $95 \%$ précédemment cité, $56 \%$ des professionnels utilisent l'écorce du tronc et de la racine pour traiter aussi bien les gastroentérites, les infections cutanées, respiratoires et sexuelles. Les feuilles macérées s'utilisent dans le traitement de la varicelle et de la rougeole. Les fruits bouillis sont consommés avec du cola (Cola nitida (Sterculiaceae)) pour traiter l'ulcère gastro-intestinale. Les graines des fruits de l'espèce sont broyées et sont données aux enfants et aux adultes comme stimulateur de l'intelligence. Le pourcentage d'utilisation de ces différentes parties (Figure 1) montre que les feuilles ou les tiges feuillées sont les plus usitées (53,9\%), suivie de l'écorce du tronc $(22,2 \%)$, des racines $(17,8 \%)$ et des fruits (6,1\%). Les formes galéniques les plus adoptées par les professionnels contactés sont le décocté (57,1\%), suivi de l'infusion (23,2\%), de la macération $(13,4 \%)$ et de la poudre $(3,8)$.

En ce qui concerne la dose, les enquêtés ont raisonné en termes de botte ou d'attache, de poignée, de cuillerée, de pincée et de verre en bambou. Pour les enfants de moins de 5 ans, la dose de la décoction ou de l'infusion 
et macération sont réparties en 3 temps (matin, midi et soir avant le coucher) et ne doit pas dépasser 2 à 5 cuillerées et $1 / 2$ verre en bambou par prise pour les adultes. Le temps d'incubation des organes frais ou séchés à l'air ambiant, et dans de l'alcool « Sodabi » varie entre 5 à 96 h selon les organes de plante concernés. Seules les personnes âgées utilisent cette forme avec le petit verre en bambou et la forme poudreuse mélangée parfois à du miel. Pour accélérer le traitement, certains thérapeutes associent à leur recette du Kaolin, de l'argile et/ou du son de maïs fermentée dans le cas des diarrhées. Pour les maux de ventre ou les crampes abdominales, le sel est ajouté pour les calmer. Dans le cas des vomissements le citron ou poivre de guinée est ajouté. En ce qui concerne la nature des aliments consommés avant les troubles, $92 \%$ des personnes contactées, laissent comprendre qu’il s’agit des plats cuisinés, suivis des aliments crus (tubercules de manioc, fruits) et l'eau. Par rapport à la fréquence et aux heures d'apparition des symptômes après un repas, les réponses varient d'une personne à une autre. Cependant, elles ont connaissance des troubles liés aux gastroentérites.

\section{Utilisations alimentaires humaines et animales et autres usages}

Les graines des fruits et les feuilles sont utilisés en alimentation. Les graines des fruits, sont surtout consommées par les personnes en âges avancés (56-75 ans) et les enfants. Les jeunes feuilles quant à elles sont consommées comme sauces légumes. Les feuilles de $P$. santalinoides sont données aux bétails pour les engraisser. Les détenteurs de connaissances des PFNL, ont rapporté que le bois du tronc est utilisé en menuiserie dans la fabrication des tabourets, des charpentes, des palettes à pâte, des cruches et des cuillères ; par les artisans pour la conception des objets d'art. Elle sert de brosse à dent dans les villages, les fleuristes utilisent ces fleurs jaunes scintillantes . L'espèce sert d'ombrage dans les formations naturelles et dans les devantures des concessions et dans les maisons. Parfois, elle sert de palissade pour les champs et les maisons des personnes à faibles moyens de survie. Les branchages et le bois du tronc sont exploités par les femmes comme bois de chauffe et pour la fabrication du charbon (tableau 5).

\section{Modes de récolte des organes et vulnérabilité de $\boldsymbol{P}$. santalinoides}

Les principaux modes de collecte des organes végétaux sont la coupure à l'aide d'une machette ou d'un coupe-coupe des rameaux, des écorces du tronc, le dessouchage des racines et l'achat des organes auprès des herboristes, le ramassage des fruits. Les feuilles ou tiges feuillées ou branchages, les écorces de tronc et de racines sont collectées durant toute l'année. Mais à des heures précises de la journée. 9 h à 12 h pour les feuilles et l'écorce de tronc, et de 6 à 9 h pour les écorces des racines. 
Tableau 5 : Récapitulatif des usages de $P$. santalinoides à des fins diverses

Secteurs

Médecine africaine

Organes utilisés

Feuilles et tiges feuillées

Ecorces du tronc

Racines et écorces des racines

Fruits

$\begin{gathered}\text { Alimentation } \\ \text { humaine }\end{gathered}$
$\begin{gathered}\text { Jeunes feuilles et/ou feuilles apicales } \\ \text { Graines des fruits de petite taille et } \\ \text { de grande taille } \\ \text { Benuiserie }\end{gathered}$

Agriculture

Energie domestique

Artisanat

Bien-être
Branchages vielles

Branchages et le bois du tronc

Bois du tronc

Branchages vielles

Bois des rameaux ; branchages vielles

Fleurs jaunes scintillantes
Traitements et/ou Intérêts

Diarrhées, dysenteries, vomissement et crampes abdominales

Variole, varicelle, infections respiratoires et sexuelle

Diarrhées, dysenteries, vomissement et crampes abdominales

Diarrhées, dysenteries, vomissement et crampes abdominales

Diarrhées, dysenteries et vomissement ; ulcère gastro-intestinale.

Légumes en sauce

Amuses gueules

Fabrication des tabourets, des charpentes, des manches de la houe, des palettes à pâte, des cruches et des cuillères Fourrage pour le bétail

Chauffage des aliments et production de charbon destiné à la vente Conception des objets d'art

Palissade pour les champs et construction d'enclos précaire

Brosse végétale ; clôture des maisons et ombrage

Conception des couronnes de fleurs

$P$. santalinoides possède un indice de vulnérabilité $\mathrm{Iv}=2,4$; soit $2 \leq \mathrm{Iv}<2,5$. Il est de ce fait identifié comme une espèce vulnérable. Les principales causes de vulnérabilité de l'espèce sont liées à la fréquence d'utilisation élevée (FU=65\%), le nombre d'usage (NU=06), le nombre d'organe végétal (05) et le mode de prélèvement des organes. En effet, le nombre très élevé du secteur d'employabilité de $P$. santalinoides accroit sa vulnérabilité.

La synthèse des informations obtenues au cours des enquêtes, révèle que l'espèce est sollicitée dans sept secteurs. Les fruits et les feuilles sont utilisés dans l'alimentation et dans la médecine africaine. La coupe perturbe la stabilité à long terme et augmente la vulnérabilité de l'espèce (tableau 4).

Tableau 4 : Paramètres de vulnérabilité de $P$. santalinoides

\begin{tabular}{cccc}
\hline Paramètres & \multicolumn{3}{c}{ Vulnérabilité à une exploitation incontrôlée } \\
\cline { 2 - 4 } & Faible (échelle = 1) & Moyenne (échelle = 2) & Forte (échelle = 3) \\
\hline Fréquence d'utilisation & Faible F.U $<20 \%$ & $\begin{array}{c}\text { Moyenne } 20 \% \\
\leq \text { F.U }<60 \%\end{array}$ & Elevée F.U $\geq 60 \%$ \\
\hline Nombre d'usage & $\mathrm{Nu}<2$ & $2 \leq \mathrm{Nu} \leq 4$ & $\mathrm{Nu} \geq 5$ \\
\hline Organe végétal utilisé & Feuille, latex & Fruit, branche & $\begin{array}{c}\text { Bois, graine, écorce, racine, } \\
\text { fleur }\end{array}$ \\
\hline Mode de collecte & Ramassage & - & $\begin{array}{c}\text { Cueillette, coupe } \\
\text { Stade de développement }\end{array}$ \\
\hline Légende : les paramètres en surbrillance sont celles considérés lors du calcul de l’indice de \\
vulnérabilité (Iv).
\end{tabular}




\section{Discussion}

\section{Connaissances endogènes et vulnérabilité de $P$. santalinoides}

La sollicitation assez élevée de l'espèce dans le traitement des gastroentérites indiquent la spécificité de ses propriétés pharmacologiques. La littérature a rapporté que l'espèce est exploitée en médecine traditionnelle pour traiter les diarrhées, les hémorroïdes, l'asthénie chez la parturiente, la dysenterie ainsi que les dystocies et pour lutter contre les helminthes (Akouègninou et al., 2006; Arbonnier, 2009). D’autres auteurs ont déjà signalé que les feuilles et les décoctés ont une grande proportion de citations endogènes (Agassounon Djikpo Tchibozo et al., 2001; Mehdiouiet Kahouadji, 2007). L'ajout du Kaolin, de l'argile et/ou du son de maïs (Zea mays) fermenté ; du sel et du citron (Citrus aurantiifolia; Rutaceae) ou poivre de guinée (Piper guineense; Piperaceae) pour traiter les gastro entérites, peut être lié aux propriétés antibiotiques des plantes médicinales d'une part et au pouvoir de rétention d'eau et de pansement des parois de l'estomac par le kaolin et l'argile d'autre part. D'après Pearce (2001), il y a une forte dépendance vis-à-vis des ressources végétales pour les besoins énergétiques ; l'utilisation du bois de $P$. santalinoides comme source d'énergie n'est pas du reste. Dans le domaine de l'alimentation, des auteurs ont évoqué l'utilisation des feuilles de Pterocarpus mildbreadii pour le bétail, une espèce du même genre que $P$. santalinoides (Akpanyung et al., 1995). Les travaux de Tian et al. (1992), sur les feuilles de cette plante ont montré la présence du magnésium, du calcium, du potassium et du phosphore. La richesse des graines des 2 morphotypes en éléments nutritionnels a été rapporté à travers d'autres études (Ayéna et Agassounon Djikpo Tchibozo, 2015). Dans le domaine de l'énergie, Keay (1989), a mentionné que le bois de l'espèce est utilisé dans la construction des charpentes. Pour le bien être, le tanin issu de la tige est utilisé en teinture (Keay, 1989).

L'action anthropique semble jouer un rôle majeur dans la destruction du couvert végétal dans le monde. Pour satisfaire leurs besoins, les populations prélèvent le bois, les écorces, les racines, les feuilles, les fruits (Traoré et al., 2011). Ainsi, les organes de $P$. santalinoides ne sont pas en marge de ce comportement. Lors des défriches, les espèces utilitaires ne sont pas épargnées des coupes, conduisant ainsi à la dégradation de la biodiversité. L'augmentation de l'effectif de la population accentue la pression anthropique exercée sur les ressources végétales. La récurrence des prélèvements affaiblit les individus ligneux et les rend vulnérables aux intempéries (vent, feux, sécheresse) et aux attaques parasitaires (Traoré et al., 2011). Ces facteurs négatifs causent la baisse de densité et la perte des peuplements végétaux. D’après Cunningham (1996), le degré de perturbation de la population d'une espèce et la vulnérabilité de cette dernière dépendent 
de la demande, de l'offre, des parties utilisées et du type de croissance de l'espèce concernée. Par ailleurs, en analysant l'impact des prélèvements de produits ou des organes végétaux, Taïta (2003), fait remarquer que les espèces dont les fruits et les graines sont consommés ou utilisés à diverses fins peuvent avoir des problèmes de dissémination. En considérant que la vulnérabilité de certaines espèces dans le Sud-Ouest de l'Ouganda s’explique par le fait qu'elles sont très sollicitées dans plusieurs domaines (énergie, construction et artisanat) dont le principal mode d'exploitation demeure une destruction partielle ou totale des individus (Cunningham, 1996), il en résulte que $P$. santalinoides est menacée, parce qu'elle est sollicitée dans sept secteurs d'activités. Ainsi, sa sollicitation dans plusieurs domaines d'utilisation à la fois peut constituer un facteur élevé de sa vulnérabilité. Par ailleurs, les usages en médecine traditionnelle qui exigent le prélèvement des écorces du tronc et des racines peuvent causer d'énormes dommages (circulation de la sève, enracinement, etc.) à cette espèce végétale. En effet, selon Hahn-Hadjali et Thiombiano (2000), l'écorçage intensif entraîne une perte de la vigueur des arbres. Cette situation provoque des troubles physiologiques au niveau de l'arbre : lesquels influencent la production des fruits qui assurent la pérennité de l'espèce. La récolte d’écorce, des tissus de la tige et des racines tue presque tous les arbres (Betti, 2001). Par contre, d'après Peters (1997), la récolte sporadique des fruits ne porte pas trop préjudice aux arbres vieux. A cet effet, les individus jeunes dont on cueille les organes végétaux sont traumatisés et sont de plus vulnérables que ceux dont on ramasse les parties tombées sur le sol. Dans l'ensemble, si la population est consciente de la dégradation des ressources végétales, elle ne perçoit pas toujours les causes de cette dégradation. Pour ce qui concerne la conservation, l'usage des branchages ou des fourches pour la palissade constitue des atouts exploitables. Les efforts de sensibilisation pour la domestication de l'espèce constituent des pistes exploitables. Cependant, la durée de conservation des fruits en étude actuellement se révèle déjà difficile à cause des insectes qui l'attaquent.

\section{Conclusion}

L’étude ethnobotanique de Pterocarpus santalinoides a permis de noter qu'il s’agit d’une espèce à usage diversifié et qu'elle est employée dans sept secteurs (médecine africaine, alimentation, agriculture, menuiserie, artisanat, énergie domestique, bien-être). Par ailleurs, cette étude a également mis en évidence sa vulnérabilité. L’indice de vulnérabilité $(I v=2,4)$ permet de classer $P$. santalinoides comme une espèce ligneuse vulnérable dans le Sud du Bénin. Des solutions envisageables sont diverses (sensibilisation, encourager les palissades raisonnées, culture drageons et graines) à travers ce travail pour la conservation de l'espèce très sollicitée. Cette espèce peut 
servir de matériel de développement endogène, si l'on arrivait à vérifier scientifiquement ses indications attribuées empiriquement, à la protéger et produire un recueil ou un manuel de données la concernant.

\section{Remerciements}

Les auteurs remercient tous ceux qui ont contribué au présent travail, en l'occurrence les professionnels de la médecine africaine béninoise contactés, les personnes détenteurs des connaissances des PNLF et "International Foundation for Science (IFS)" qui avec l'organisation pour l'interdiction des armes chimiques (OPCW) sous le grantn ${ }^{\circ} \mathrm{F} / 5550-1$ ont cofinancé l'étude.

\section{References:}

Adomou AC., Agbani OP., Sinsin B., 2011. Plantes In Protection de la Nature en Afrique de l'Ouest : Une Liste Rouge pour le Bénin. Nature Conservation in West Africa: Red List for

Benin, Neuenschwander P., Sinsin B., Goergen G. (eds). International Institute of Tropical Agriculture. Ibadan, Nigeria, 21-46.

Adoukonou-Sagbadja H., Dansi A.,Vodouhè R., Akpagana K., 2006. Indigenous knowledge and traditional conservation of Fonio millet (Digitaria exilis Stapf, Digitaria iburua Stapf) in Togo. Biod.. Conserv., 15: 2379-2395.

Agassounon Djikpo Tchibozo M., Anani KT., Ameyapoh Y., Toukourou F., de Souza C., Gbéassor M., 2001. Evaluation de la qualité hygiénique de six plantes médicinales et des phytomédicaments traditionnels. Pharm. et Méd. Tradit. Afr.,6(11): 83-92.

Agassounon Djikpo Tchibozo M., Ayi-Fanou L., Oumorou M., Mensah GA., Agbangla C., Ahanhanzo C., de Souza C., 2012a. Usages thérapeutiques traditionnels de Flacourtiaindica (Burm F.) Merr (Flacourtiaceae) et de Rytigynia canthioides (Benth.) Robyns (Rubiaceae), deux espèces de la flore béninoise. J. Rech. Sci. Univ. Lomé (Togo), Série A, 14(1): 53-61.

Agassounon Djikpo Tchibozo M., Savadogo A., Karou DS., Toukourou F., de Souza C., 2012b. Connaissances endogènes et études phytochimiques de Flacourtia flavescens Willd. (Flacourtia indica (Burm f.) Merr.). Tropicultura, 30: 1, 3-8.

Akoègninou A., Burg Van Der WJ., Maesen Van Der LJG., Adjakidje V., Essou JP., Sinsin

B., Yedomonhan H., 2006. Flore Analytique du Bénin. Backuys Publishers, $1034 \mathrm{p}$.

Assogbadjo AE., Kyndt T., Sinsin B., Gheysen G., Van Damme P., 2006. Patterns of genetic and morphometric diversity in baobab (Adansonia 
digitata L.) populations across different climatic zones of Benin (West Africa). Ann Bot 97: 819-830

Assogbadjo AE., Sinsin B., Codjia JTC., Van Damme P., 2005a. Ecological diversity and pulp, seed and kernel production of the baobab (Adansonia digitata) in Benin. Belg J Bot 138(1): 47-56.

Assogbadjo AE., Sinsin B., Van Damme P., 2005b. Caractères morphologiques et production des capsules de baobab (Adansonia digitata L.) au Bénin. Fruits 60(5): 327-340.

Ayéna C. A., Agassounon Tchibozo M., Adoukonou-Sagbadja H., Karou D. S., Anani K., de Souza C., 2014. Activités antimicrobiennes des extraits de trois plantes médicinales utilisées dans le traitement traditionnel des toxiinfections alimentaires au Sud du Bénin. J. Rech. Sci. Univ. Lomé (Togo), Série A, 16(3): 23-31.

Ayéna C. A., Agassounon Djikpo Tchibozo M., 2015 :Valorisation de Pterocarpus santalinoides L'Hér. ex De. (Papilionoideae), une plante alimentaire et médicinale utilisée au Bénin en Afrique de l’Ouest. J. of Appl. Biosc., 90: 8377- 8386.

Belem B., Sanou-Nana P., 2009. Plantes médicinales utilisées pour le soin des enfants dans la ville de Ouagadougou, Burkina Faso, Afrique de l'Ouest. Le Flamboyant, 65: 9 -12.

Benkhnigue O., Zidane L., Fadli M., Elyacoubi H., Rochdi A., Douira A., 2011. Etude ethnobotanique des plantes médicinales dans la région de Mechraâ Bel Ksiri (Région du Gharb du Maroc. Acta Bot. Barc., 53: 191216

Betti JL., 2001. Vulnérabilité des plantes utilisées comme antipaludiques dans l'arrondissement de Mintom au Sud de la réserve de Biosphère du Dja (Cameroun). Syst. Geogr. Pl., 71: 661- 678.

Bognon C., 1991. Notes ethnobotaniques sur la médecine traditionnelle en pays Wè (Côte d'Ivoire) : quelques problèmes méthodologiques. Revue Méd. Pharm. Afr., 5(1): 55-62.

Codjia JTC., Assogbadjo AE., Ekue' MRM., 2003. Diversité et valorisation au niveau local des ressources forestières alimentaires végétales du Bénin. Cahiers Agric 12: 321-331

Cunningham AB., 1996. Peuples, Par ces Plantes. Recommandation pour les zones à usages multiples et les alternatives de développement autour du parc national de Bwindi Impénétrable. Ouganda. Document de travail Peuples et Plantes n4. UNESCO ; Paris, p.64.

Cuq J. L., 1997. Microbiologie alimentaire : Maladies microbiennes transmis par les aliments. Sciences et Techniques. Montpellier, 129p.

Dagnelie P., 1998. Statistiques théoriques et appliquées. Statistique descriptive et bases de l'influence statistique Paris et Brussels: De Boeck et Larcier. 508 p. 
Hahn-Hadjali K., Thiombiano A., 2000. Perception des espèces en voie de disparition en milieu Gourmantché (Est du Burkina Faso). Berichte des Sonderforschungsbereichs, 268, Band 14, Frankfurt, 285-297.

KeayRW., 1989. Arbres de Nigéria. Claredon Press, Oxford, USA, pp1-450

Kristensen M., Balslev H., 2003 : Perceptions, use and availability of woody plants among the Gourounsi in Burkina Faso. Biod. and Conserv., 12: 17151739.

Lykke AM., Kristensen MK.,Ganaba S., 2004. Valuation of local use and dynamics of 56 woody species in the Sahel. Biod. and Conserv., 13: 19611990.

Mayling SH., 1983. Méthodologie d'enquête socioculturel pour des alimentations en eau et assainissement; Groupe Consultatif pour la Technologie (TAG), Note technique $\mathrm{N}^{0} 1$ du TAG Washington, D.C.20433, USA, pp. 5-12.

Mehdioui R., Kahouadji A., 2007. Etude ethnobotanique auprès de la population riveraine de la forêt d'Amsittène : cas de la Commune d'Imi n’Tlit (Province d'Essaouira). Bulletin de l'Institut Scientifique, Rabat, section Sciences de la Vie, 29: 11-20.

Pearce DW. 2001. The Economic Value of Forest Ecosystems. Ecosy. Health, 7(4): 284-296.

Peters CM., 1997. Exploitation Soutenue de Produits Forestiers autres que le Bois en Forêt Tropicale Humide: Manuel d'Initiation Ecologique. Série générale du Programme d'Appui à la Biodiversité 2: 49p. WWW-NCWRI/USAID.

Taïta P., 2003. Use of woody plants by locals in Mare aux Hippopotames Biosphere Reserve in western Burkina Faso. Biodiv. and Conserv., 12: 12051217.

Tian G., Kang BT., Buissaard L., 1992. Effects of chemical composition on $\mathrm{N}$, Ca and $\mathrm{Mg}$ release during incubation of leaves from selected agroforestry and fallow plant species. Biogeochem., 16(2): 103-119.

Traoré L., Ouédraogo I., Ouédraogo A., Thiombiano A., 2011. Perceptions, usages, et vulnérabilité des ressources végétales ligneuses dans le Sud-Ouest du Burkina-Faso. Int. J. Biol. Chem. Sci., 5(1): 258-278. 